\title{
Caractérisation Physico-Chimique des Sols des Vallées Agricoles de la Commune de Ziguinchor au Sénégal
}

\author{
Maurice Dasylva, \\ ( $\mathrm{PhD}$ en Sciences Agronomiques et Environnement) \\ Université Assane Seck de Ziguinchor, Sénégal \\ Ngor Ndour, \\ (Maître-Assistant, PhD, Botaniste Environnementaliste) \\ Université Assane Seck de Ziguinchor, Sénégal \\ Mamadou Abdoul Ader Diédhiou, \\ ( $\mathrm{PhD}$ en Sciences Agronomiques et Environnement) \\ Université Assane Seck de Ziguinchor, Sénégal \\ Bienvenu Sambou, \\ (Maître de Conférences, PhD, Environnementaliste) \\ Université Cheikh Anta Diop de Dakar, Sénégal
}

Doi: 10.19044/esj.2019.v15n15p165 URL:http://dx.doi.org/10.19044/esj.2019.v15n15p165

\section{Résumé}

Bien qu'elle demeure un axe stratégique de lutte contre la pauvreté, l'agriculture urbaine est peu considérée par les décideurs et souffre des mutations spatiales et pédologiques à Ziguinchor. Cette étude vise de de façon générale à établir un cadre de références des caractéristiques physicochimiques des sols des vallées agricoles de la ville de Ziguinchor. Elle a été réalisée sur la base de 180 carottes de sols collectés à l'horizon [0-20] $\mathrm{cm}$. Elles ont été constituées en 12 échantillons composites analysés par l'Institut Nationale de Pédologie du Sénégal. L'étude a permis de noter une prédominance de sols hydromorphes à texture limono-argilo-sableuse, caractérisés par une structure relativement stable. Bien qu'ils soient d'une manière générale acides $(\mathrm{pH} \leq 5)$ et sodiques par endroit, ces sols sont relativement riches en $\mathrm{MO}(0,84 \%)$, particulièrement dans les parties amont des vallées. La vallée de Kandialang-Djibock présente des sols plus riches en MO $(1,143 \%)$ et un pourcentage de cations nutritifs disponible pour les cultures qui est estimé à environ $80 \%$. Cependant, dans l'ensemble des vallées, les sols sont considérés très pauvre en $\mathrm{N}$ avec une teneur comprise dans l'intervalle [0,10-0.25\%], bien que le rapport $\mathrm{C} / \mathrm{N}$ soit inférieur à 12 . L'urbanisation, l'ensablement, l'acidification et la salinisation progressive 
des sols constituent les principales contraintes de la production agricole. La solution à ces contraintes repose sur le maintien de la fertilité de ces sols par la réhabilitation des digues anti-sel et la lutte contre l'ensablement des vallées.

Mots-clés : Sol, Vallée agricole, Caractéristiques physico-chimiques, Contraintes

\title{
Physico-Chemical Characterization of the Agricultural Valleys' Soils of the Municipality of Ziguinchor in Senegal
}

\author{
Maurice Dasylva, \\ ( $\mathrm{PhD}$ en Sciences Agronomiques et Environnement) \\ Université Assane Seck de Ziguinchor, Sénégal \\ Ngor Ndour, \\ (Maître-Assistant, $\mathrm{PhD}$, Botaniste Environnementaliste) \\ Université Assane Seck de Ziguinchor, Sénégal \\ Mamadou Abdoul Ader Diédhiou, \\ (PhD en Sciences Agronomiques et Environnement) \\ Université Assane Seck de Ziguinchor, Sénégal \\ Bienvenu Sambou, \\ (Maître de Conférences, $\mathrm{PhD}$, Environnementaliste) \\ Université Cheikh Anta Diop de Dakar, Sénégal
}

\begin{abstract}
Despite the importance of urban agriculture as a strategic axe of resilience to poverty, Ziguinchor's urban agriculture is not well considered by the municipalty autorities and suffers from spatial and soil transformations. The study aims to establish a frame of reference for soil physicochemical characteristics of the agricultural valleys of Ziguinchor's city. For that, 180 soil core samples were collected on the horizon [0-20] $\mathrm{cm}$. They were made up of 12 composite samples analyzed at the Senegal National Pedology Institute. The study showed a predominance of hydromorphic soils with a silty-clay-sandy texture, characterized by a relatively stable structure. Although generally acidic $(\mathrm{pH} \leq 5)$ and sodic by location, these soils are relatively rich in $\mathrm{OM}(0.84 \%)$, particularly in the upstream parts of the valleys. The Kandialang-Djibock valley has the most rich soils in OM
\end{abstract}


$(1.143 \%)$ and a nutrient cation percentage available for crops that exceeds $80 \%$. However, in all valleys, the soils are considered to be very low in $\mathrm{N}$ with an interval content [0,10-0.25\%], although the $\mathrm{C} / \mathrm{N}$ ratio is less than 12 . Urbanization, silting, acidification and gradual salinization of soils are the main constraints of agricultural production. The solution to these constraints lies in maintaining the fertility and agricultural productivity of these soils, rehabilitating anti-salt dykes and combating the silting of valleys.

Keywords: Soil, Agricultural valley, Physico-chemical characteristics, Constraints

\section{Introduction}

L'agriculture urbaine est, d'une manière générale, associée à la culture des produits essentiellement destinés à l'alimentation des habitants d'une ville (Hista, 2007 ; Aubry, 2012 ; Duchemin, 2012). Elle est considérée comme étant un axe stratégique de développement urbain en raison des divers services environnementaux et socioéconomiques qu'elle procure particulièrement aux villes des pays en développement (Kakai et al., 2010 ; Ba et al., 2016 ; Bagré et al., 2002 ; Sueur, 2011). L'agriculture urbaine est pratiquée par 800 millions de citadins et fournit $15 \%$ des denrées alimentaires mondiales (FAO, 2010). A Ziguinchor, elle contribue à la lutte contre la pauvreté et reste un gage de la sécurité alimentaire. Les revenus moyens annuels d'un producteur des plantes aromatiques et médicales sont évalués à 207462 FCFA et représentent 43\% de ceux d'un ménage agricole (Dasylva et al. 2018). La production rizicole autoconsommée assure 9 à plus de12 mois de subsistance à 38,2\% des ménages riziculteurs (Dasylva, 2018). Cependant, bien que l'agriculture urbaine demeure un facteur de résilience à la pauvreté à Ziguinchor, elle souffre de sa non prise en compte dans le plan local de développement (Dasylva et $a l ., 2017$ ). Les vallées de la ville, bien que non aedificandi, subissent l'urbanisation galopante qui se traduit par l'occupation progressive et anarchique de leur bassin versant par le bâti. A cette contrainte de l'agriculture urbaine, s'ajoute l'avancée de la langue salée dans l'espace rizicole frontale au fleuve Casamance suite au manque d'entretien des digues de protection par les producteurs. D'ailleurs, 8,73\% des 838,9 ha que couvrent ces vallées ne sont plus exploitées en raison de l'effet conjugué de la salinisation et de l'acidification des sols (Dasylva, 2018). Les rares études agro-environnementales, réalisées dans la ville de Ziguinchor (Keita, 2013 ; Dasylva ,2013 ; Sow, 2014, Dasylva et al., 2017) ne traitent pas souvent des caractéristiques pédologiques de ces systèmes d'exploitation agricole. Pourtant, de telles études pourraient contribuer à l'établissement d'un cadre de référence pédologique de ces sols et à la réalisation de diagnostics de l'agriculture urbaine à Ziguinchor. La problématique ainsi posée justifie ce 
présent article qui porte sur la caractérisation physico-chimique des sols des vallées agricoles de la commune de Ziguinchor.

\section{Materiel et Methodes}

\section{Présentation du site de l'étude}

La commune de Ziguinchor, site de l'étude, a été créée en 1888 et est actuellement le chef-lieu administratif de la région. Située au Sud-Ouest du Sénégal, Cette commune est localisée entre le méridien $16^{\circ}$ et $17^{\circ}$ et les parallèles $12^{\circ}$ et $13^{\circ}$ (Figure 1). Elle est caractérisée par un climat de type soudano-côtier Sud (Sagna, 2005). La pluviométrie moyenne annuelle est évaluée, entre 1981 et 2017, à 1310,54 mm. Malgré la variabilité pluviométrique, Ziguinchor est l'une des villes les plus arrosées du Sénégal. Cette bonne pluviométrie corrélée à l'étendue des dépressions offre à cette ville un grand potentiel agronomique.

Selon les projections démographiques de l'ANSD $^{2}$ (2015) la ville de Ziguinchor aurait une population de 232217 habitants en 2017. C'est une ville cosmopolite composée essentiellement de diolas $(35 \%)$ et de mandingues (18\%). Les ménages agricoles représentent 26\% ( $\left.\mathrm{PAM}^{3}, 2012\right)$. Elles pratiquent la riziculture pluviale et le maraîchage de contre-saison dans les vallées périurbaines.

${ }^{2}$ Agence Nationale de la Statistique et de la Démographie

${ }^{3}$ Programme Alimentaire Mondial 


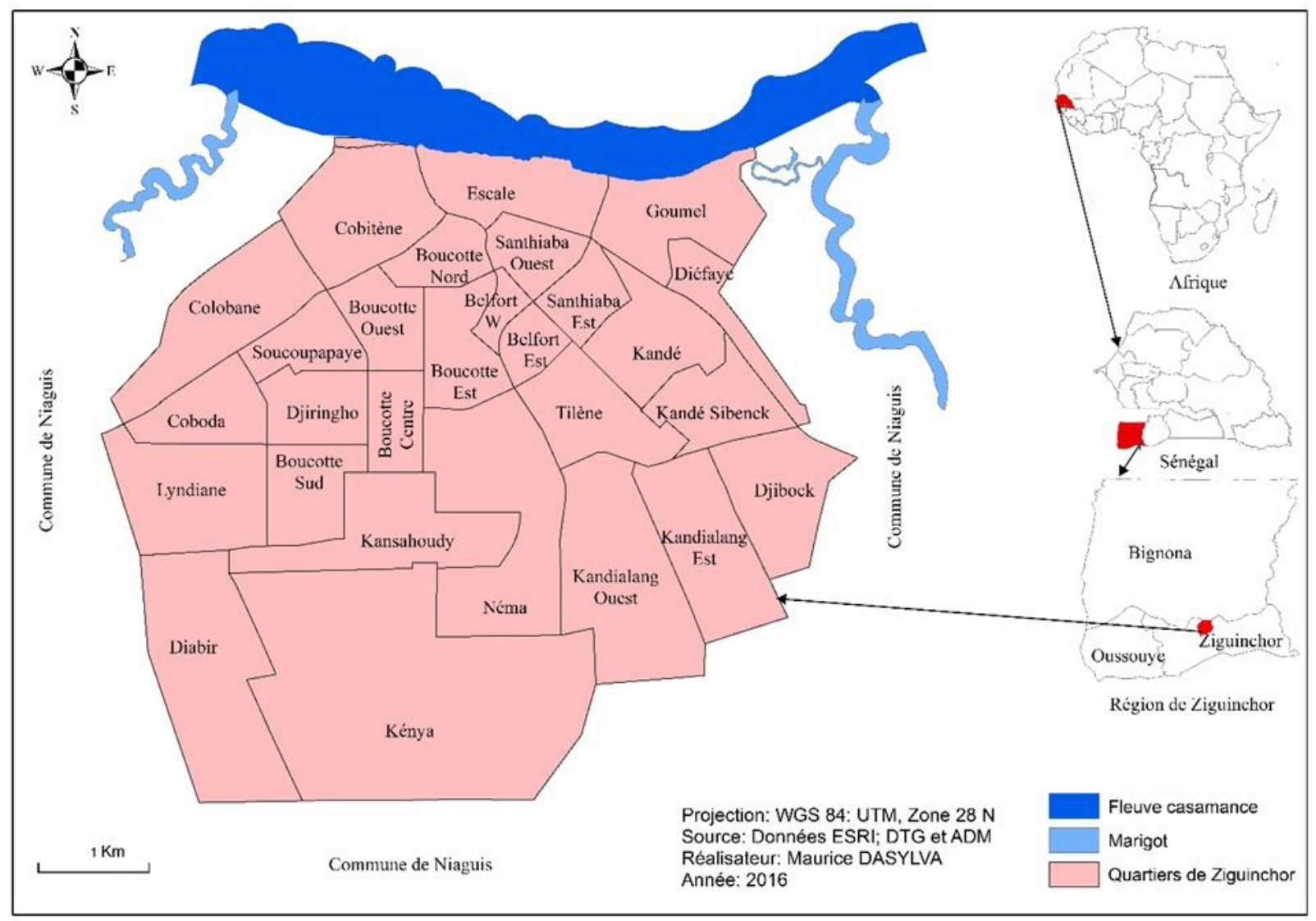

Fig. 1 : Carte de localisation de la commune de Ziguinchor

\section{Choix des vallées et des sites de prélèvement des sols}

La prospection réalisée dans la ville a permis de noter les vallées agricoles et d'identifier les sites à retenir pour la collecte des carottes de sols. Le choix des vallées a porté sur celles qui jouxtent les quartiers de Diéfaye, Cobitène-Colobane, Lyndiane-Diabir et Kandialang-Djibock où l'essentiel des activités rizicoles et maraîchères sont pratiquées.

Dans chacune de ces 4 vallées, 9 casiers rizicoles ont été identifiés et localisés à l'aide d'un GPS ${ }^{4}$ suivant la toposéquence « terre ferme-fleuve Casamance». Ainsi, trois (3) casiers ont été choisis dans chacune des parties amont, médiane et aval de chaque vallée (Figure2). Une telle répartition des casiers dans l'espace vise une meilleure prise en compte de la probabilité de la micro-variabilité des caractéristiques physico-chimiques des sols en fonction de la toposéquence.

\footnotetext{
${ }^{4}$ General Positioning System
} 


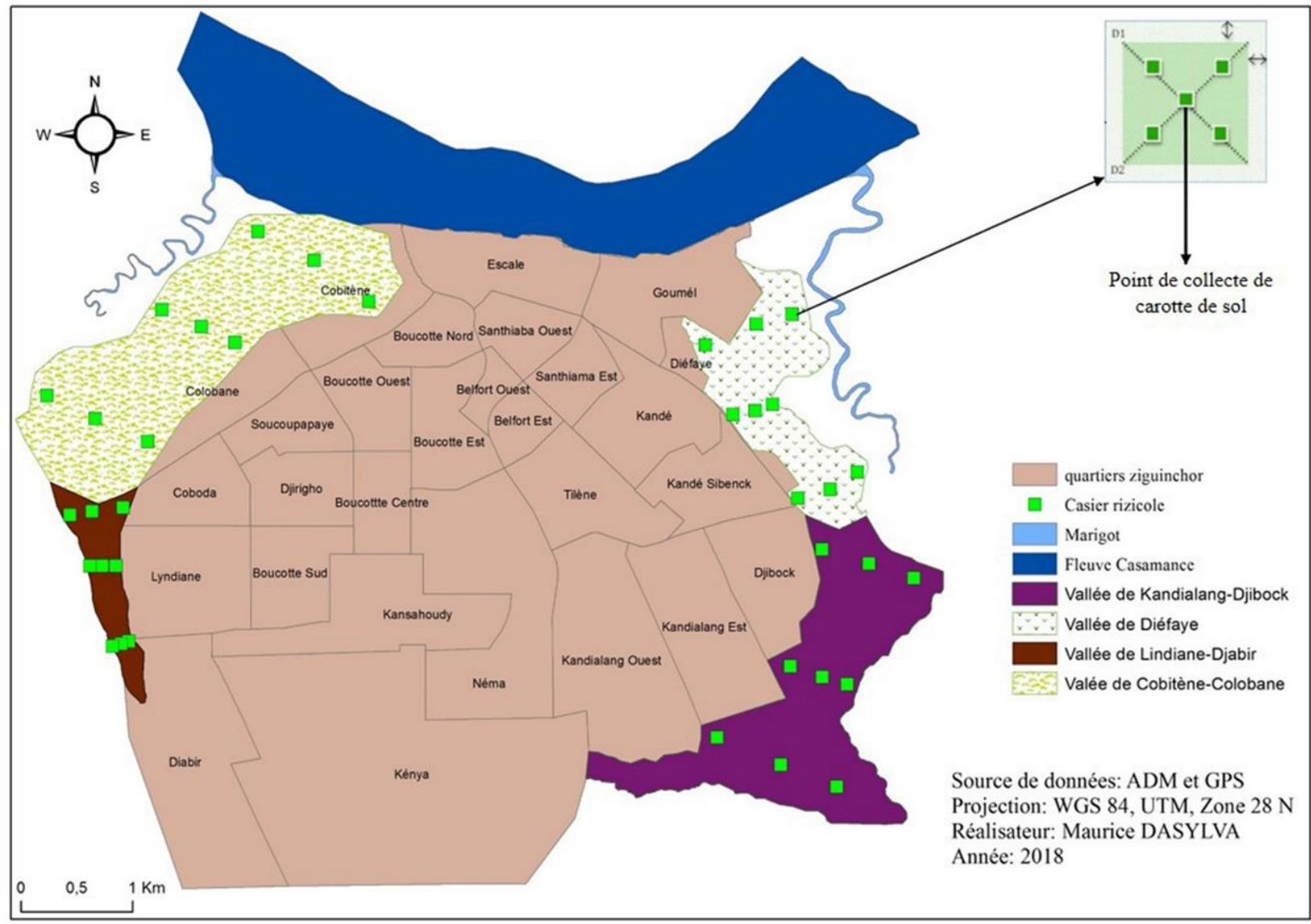

Figure 2 : Localisation des casiers de collecte des carottes de sols dans les vallées agricoles de Ziguinchor

\section{Prélèvement de carottes de sols}

L'étude s'est intéressée à la caractérisation physico-chimique des sols des rizières en vue de déterminer leur potentiel agronomique. A cet égard, la granulométrie, le carbone, l'azote total, la capacité d'échange cationique, le $\mathrm{pH}$, la salinité et la stabilité structurale ont été déterminés à partir de la méthode de Faraoun et Bénabdèli (2010).

Dans chacun des 9 casiers rizicoles localisés dans chaque vallée, 5 carottes de sols sont prélevés suivant les diagonales; soit une carotte dans chacun des 4 côtés du casier rizicole et le cinquième au point d'intersections de ses diagonales (Figure 2). Au total, 180 carottes de sols ont été prélevées, à raison de 15 carottes par niveau de toposéquence soit 45 carottes pour chacune des quatre vallées. Les carottes de sol ont été collectées dans l'horizon [0 - 20] $\mathrm{cm}$ à l'aide d'une tarière pédologique. Le choix de cet horizon pédologique a tenu compte de la profondeur d'occurrence des racines des cultures de riz et de leur alimentation en chaleur, eau et éléments nutritifs 
(Reyniers et al., 1979 ; Koné et al., 2008 ; Touhtouh et al., 2014 ; Rafik et al., 2015). Les producteurs rizicoles choisis ont été identifiés grâce à la collaboration des chefs et/ou délégués de quartier. Ils ont fait l'objet d'entretien structuré sur les pratiques culturales et les techniques de fertilisation des sols.

\section{Composition des échantillons de sol}

Les carottes de sols prélevées ont subi deux niveaux d'association pour avoir des échantillons composites pour chaque vallée. Le premier niveau a eu lieu à l'échelle du casier rizicole où les 5 carottes des sols prélevées ont été mélangées puis l'échantillon composite obtenu a été ensaché et codé. Ainsi, pour chaque vallée, on a constitué trois échantillons composites dans sa partie en amont, 3 dans sa partie médiane et 3 autres dans sa partie en aval. Le deuxième niveau de constitution des échantillons a été réalisé au Laboratoire d'Agroforesterie et d'Ecologie (LAFE) de l'Université Assane Séck de Ziguinchor. Il a consisté, pour chaque vallée, à mélanger les 3 échantillons de sols de chaque niveau de toposéquence en un échantillon composite donnant ainsi 1 échantillon par niveau de toposéquence et 3 échantillons par vallée. Soit 12 échantillons ensachés et étiquetés au total.

\section{Analyse des sols}

Les 12 échantillons de sols ont été envoyés à l'Institut National de Pédologie (INP) du Sénégal. Ils ont été analysés suivant le protocole technique de cette institution. La texture des sols a été évaluée à l'aide de la grille de classification de la FAO-UNESCO (1975). La texture des différents échantillons de sol a été déterminée à l'aide du triangle textural de Tavernier et Maréchal (1958) cité par Rafik et al. (2015), suivant l'approche méthodologique de Masson (2012). Les valeurs de $\mathrm{pH}$ des sols ont été interprétées à l'aide de la légende des séries de sol proposée par l'INP ${ }^{5}$ (1992). L'analyse du degré de salinité des sols a été basée sur l'interprétation de leur conductivité électrique (CE) suivant les normes de Durand (1983). Le niveau de saturation des sols en Carbone (C), Matière organique (MO) et Azote $(\mathrm{N})$ a été analysé en utilisant l'échelle d'interprétation du CIRAD et du GRET (2002). Le rapport C/N utilisé pour apprécier l'activité biologique du sol (Ramade, 2008) a été établi. La teneur des sols en Phosphore (P) assimilable, en bases échangeables [Calcium (Ca), Magnésium ( $\mathrm{Mg})$, Potassium $(\mathrm{K})$ et Sodium (Na)] ainsi que la Capacité d'Echange Cationique (CEC.) et le Taux de saturation du sol (TS) ont été analysés à l'aide de l'échelle d'interprétation de Landon (1984).

\footnotetext{
${ }^{5}$ Institut National de Pédologie du Sénégal
} 


\section{Analyses statistiques}

Les analyses statistiques ont été réalisées à l'aide du Logiciel XLSTAT Pro 2014.5.03. L'Analyse en Composante Principale (ACP), associée au test de sphéricité de Bartlett a permis de noter les corrélations entre les variables physicochimiques des sols. Le test de comparaison de Kruskal-Wallis a été réalisé pour déterminer le niveau de différence de la tendance moyenne des variables caractéristiques des sols entre les trois niveaux de toposéquence (amont, médian et aval) de chaque vallée d'une part et entre les vallées d'autre part. Les tests ont été réalisés au seuil de signification alpha $=0,05$. La différence de la variation de la tendance moyenne d'une variable suivant un facteur est significative lorsque la p-value est inférieure à Alpha (5\%).

Des modélisations de régressions linéaires entre les variables qui ont des corrélations significatives ont été réalisées et celles ayant la plus grande valeur du coefficient de détermination $\left(\mathrm{r}^{2}\right)$ ont été retenues. Ce $\mathrm{r}^{2}$ exprime la proportion centésimale qu'une ou des variable(s) déterminante (s) explique(nt) celle(s) dépendante(s).

\section{Resultats}

\section{Granulométrie et texture des sols}

Le Tableau 1 présente la teneur des différentes fractions granulométriques et la textures des sols. Son analyse montre qu'il n'existe pas de différence significative entre les teneurs moyennes des fractions de sols des différentes vallées $(p>0,05)$.

Tableau 1 : Les constituants physiques des sols des vallées de la ville de Ziguinchor

\begin{tabular}{llcccc}
\hline \hline \multirow{2}{*}{ Vallée } & \multirow{2}{*}{ Partie } & \multicolumn{3}{c}{ Granulométrie } & \multirow{2}{*}{ Texture } \\
\cline { 3 - 5 } & & $\mathbf{A} \%$ & L\% & $\mathbf{S \%}$ & \\
\hline \hline \multirow{2}{*}{ Cobitène-Colobane } & Amont & 28,50 & 25,56 & 45,94 & Las. \\
& Médiane & 21,50 & 26,75 & 51,75 & Las. \\
& Aval & 19,75 & 19,53 & 60,72 & Ls. \\
& Moyenne & $\mathbf{2 3 , 2 5}$ & $\mathbf{2 3 , 9 5}$ & $\mathbf{5 2 , 8 0}$ & Las. \\
\hline Diéfaye & Amont & 15,50 & 16,30 & 66,20 & Ls. \\
& Médiane & 17,50 & 9,42 & 75,08 & Ls. \\
& Aval & 46,00 & 37,45 & 16,55 & A. \\
& Moyenne & $\mathbf{2 6 , 3 3}$ & $\mathbf{2 1 , 0 6}$ & $\mathbf{5 2 , 6 1}$ & Las. \\
\hline Kandialang-Djibock & Amont & 25,00 & 31,74 & 43,26 & L. \\
& Médiane & 27,25 & 23,49 & 49,26 & Las. \\
& Aval & 16,50 & 17,12 & 66,38 & Ls. \\
& Moyenne & $\mathbf{2 2 , 9 2}$ & $\mathbf{2 4 , 1 2}$ & $\mathbf{5 2 , 9 6}$ & Las. \\
\hline Lyndiane & Amont & 19,75 & 27,84 & 52,41 & Las. \\
& Médiane & 21,00 & 30,38 & 48,82 & Las. \\
& Aval & 44,50 & 6,68 & 48,63 & As. \\
& Moyenne & $\mathbf{2 8 , 4 2}$ & $\mathbf{2 1 , 6 3}$ & $\mathbf{4 9 , 9 5}$ & Las. \\
\hline \hline
\end{tabular}




\begin{tabular}{lllll}
\multirow{2}{*}{ Test Kruskal-Wallis } & P-value & 0,813 & 0,74 & 0,84 \\
& Alpha & 0,050 & 0,05 & 0,05 \\
\hline \hline
\end{tabular}

Las : Limono-argilo-sableux ; Ls : Limono-sableux ; L : Limoneux ; As : Argilo-sableux, A : argileux

La teneur en sable est moyennement égale dans les différentes vallées avec des valeurs proches de 50\%. Elle baisse d'amont en aval dans les vallées de Cobitène-Colobane et Kandialang-Djibock. Par contre, dans les vallées de Diéfaye et Lyndiane, la teneur en sable en aval est 2 à 2,6 fois plus importante que celle de l'amont et de la médiane.

La proportion de limons est beaucoup plus importante dans les vallées de Kandialang-Djibock $(24,12 \%)$ et Cobitène-Colobane (23,95\%). Dans la vallée de Cobitène-Colobane, la fraction limoneuse est quasi équitablement répartie. Par contre, dans celle de Diéfaye, la fraction limoneuse est plus présente en aval $(37,45 \%)$ où elle est 2 à 3 fois plus importante que les valeurs de l'amont et de la médiane. Dans la vallée de Kandialang-Djibock, la fraction limoneuse est plus importante en amont $(31,74 \%)$ et dans la partie médiane (23,49\%). Dans la vallée de Lyndiane, les teneurs en limon de l'amont et de la médiane sont environ 4 fois plus importantes que celles de l'aval.

La vallée de Lyndiane présente la plus importante teneur moyenne en argile $(28,42 \%)$ suivie de celle de Diéfaye $(26,33 \%)$. Les vallées de CobitèneColobane et Kandialang-Djibok ont les plus faibles teneurs en argile. Cette fraction de sol est quasi équitablement répartie suivant le gradient de toposéquence dans les vallées de Cobitène-Colobane, Kandialang-Djibock et Lyndiane. Par contre, dans la vallée de Diéfaye, la fraction sable est beaucoup plus concentrée en amont et en médiane et représente 4 fois celle de l'aval.

L'analyse texturale a fait ressortir, de manière globale, une prédominance des sols limono-argilo-sableux dans les vallées. Les sols des vallées de Cobitène-Colobane et Lyndiane ont une texture identique à caractère limono-argilo sableux au niveau de leurs parties amont et médiane. Ils sont limono-sableux dans la partie de la vallée de Cobitène-Colobane et argilo-sableux dans celle de Lyndiane. Quant à la vallée de Diéfaye, ses sols présentent un dimorphisme textural à caractère limono-sableux en amont et en zone médiane et argileux en aval. Par contre, la vallée de Kandialang-Djibock a des sols à caractère textural distinct dans chacune de ses parties. Ils sont strictement limoneux en aval, limono-argileux sableux dans la zone médiane et argilo-sableux en aval.

\section{Propriétés acido-basiques et salinité des sols}

Le $\mathrm{pH}$ et la conductivité électrique (CE) des sols analysés présentent de légères variabilités suivant les vallées et la toposéquence (Tableau 2). 
Tableau 2: Variabilité du $\mathrm{pH}$ et de la $\mathrm{CE}$ des sols dans les vallées de la ville de Ziguinchor

\begin{tabular}{llcc}
\hline Vallée & Parties & pHeau 1/ 2,5 & CE 1/ 10 en $\boldsymbol{\mu S / C m}$ \\
\hline \hline Cobitène-Colobane & Amont & 6,8 & 54 \\
& Médiane & 5 & 74 \\
& Aval & 5 & 83 \\
& Moyenne & $\mathbf{5 , 6 0}$ & $\mathbf{7 0 , 3 3}$ \\
& Amont & 5,6 & 63 \\
Diéfaye & Médiane & 5,7 & 92 \\
& Aval & 5,1 & 240 \\
& Moyenne & $\mathbf{5 , 4 7}$ & $\mathbf{1 3 1 , 6 7}$ \\
& Amont & 6,7 & 28 \\
& Médiane & 6,3 & 22 \\
Kandialang Djibock & Aval & 7,8 & 70 \\
& Moyenne & $\mathbf{6 , 9 3}$ & $\mathbf{4 0 , 0 0}$ \\
& Amont & 6,7 & 77 \\
& Médiane & 5 & 26 \\
Lyndiane & Aval & 5 & 26 \\
& Moyenne & $\mathbf{5 , 5 7}$ & $\mathbf{4 3 , 0 0}$ \\
& P-value & 0,293 & 0,182 \\
& Alpha & 0,050 & 0,050 \\
\hline \multirow{2}{*}{ Test Kruskal-Wallis } & & &
\end{tabular}

L'analyse du Tableau 2 permet de noter que les sols des vallées de Cobitène-Colobane et Lyndiane présentent le $\mathrm{pH}$ le plus faible et sont considérés potentiellement acides. Du point de vue de la toposéquence, les sols de ces deux vallées ont un $\mathrm{pH}$ relativement neutre en amont $(\mathrm{pH}=[6,7-6,8])$ et très acides dans leurs parties médiane et aval. Dans la vallée de KandialangDjibock, les sols ont un $\mathrm{pH}$ neutre $(\mathrm{pH}=6,7)$ en amont, légèrement acide $(\mathrm{pH}=6,3)$ dans la zone médiane et légèrement alcalin $(\mathrm{pH}=7,8)$ en aval. Dans la vallée de Diéfaye, les sols sont modérément acides avec un $\mathrm{pH}=5,6$ et 5,7 respectivement en amont et en zone médianes et très acides $(\mathrm{pH}=5,1)$ en aval.

La conductivité électrique des sols des vallées agricoles est très faible. Elle est évaluée, en moyenne, à 131,67 $\mu \mathrm{S} / \mathrm{cm}$ dans la vallée de Diéfaye, 70,33 $\mu \mathrm{S} / \mathrm{cm}$ dans la vallée de Cobitène-Colobane, $43,00 \mu \mathrm{S} / \mathrm{cm}$ dans la vallée de Lyndiane et 40,00 $\mu \mathrm{S} / \mathrm{cm}$ dans celle de Kandialang-Djibock. Dans certains des casiers rizicoles abandonnés, la conductivité électrique est relativement élevée (3999 $\mu \mathrm{S} / \mathrm{cm})$ traduisant ainsi un important taux de salinité de ces sites.

\section{Teneur en éléments fertilisant des sols}

Le tableau 3 présente les teneurs obtenues en Carbone, en Matière organique, en Azote, en Phosphore assimilable, en bases échangeables ainsi qu'en Capacité d'échange cationiques et en Taux de saturation des sols. L'étude permet de noter qu'il n'existe aucune différence significative entre les 
teneurs moyennes des éléments fertilisants enregistrées dans les différentes vallées au seuil alpha (5\%). L'analyse de ce tableau montre que la teneur moyenne en $\mathrm{C}$ est plus importante dans la vallée de Kandialang-Djibock. Elle est égale à 1,044\% dans sa partie médiane et atteint environ 2 fois cette teneur en amont et en aval. Dans la vallée de Lyndiane, la teneur moyenne en $\mathrm{C}$ en amont représente presque le double de celle qui a été notée dans la partie médiane et en aval. Les vallées de Diéfaye et Cobitène-Colobane sont moins fournies en C. Dans la vallée de Diéfaye, on constate une migration du $\mathrm{C}$ vers l'aval $(0,808 \%)$ où elle représente environ 2 fois celle notée en amont et 4 fois celle notée dans la partie médiane. Par contre, dans la vallée de CobitèneColobane, l'amont concentre la plus grande teneur en carbone $(0,532)$ qui représente 3 fois celle de sa partie médiane et 5 fois celle de l'aval.

La concentration en MO des sols suit la même logique de variabilité que celle du C. La vallée de Cobitène-Colobane est globalement pauvre en MO avec une teneur moyenne de $0,453 \%$. Sa partie amont est moyennement riche en MO $(0,917 \%)$ alors que ses parties médianes et aval sont considérées très pauvres en MO. Quant à la vallée de Diéfaye, les sols sont moyennement riches en MO avec une teneur moyenne de 0,792\%. Toutefois, sa partie médiane est considéré très pauvre en $\mathrm{MO}$, celle amont en est pauvre alors que celle aval en est riche. Dans la vallée de Lyndiane, les sols sont, d'une manière générale, moyennement richesses en MO (0,985\% en moyenne). Ceux de ses parties médiane et aval ont une richesse en MO moyenne, avec des teneurs respectives de $0,747 \%$ et $0,917 \%$. Cependant, sa partie amont est considérée riche en MO (1,290\%). La vallée de Kandialang-Djibock a, d'une manière globale, des sols présentant une meilleure richesse en $\mathrm{MO}$, avec une teneur moyenne estimée à $1,143 \%$. Ceux en amont et en aval, avec respectivement une teneur de $0,781 \%$ et $0,849 \%$, sont estimés moyennement riches en MO. Sa partie médiane est estimée riche en MO (1,800\%). L'étude a permis de noter que les sols des vallées agricoles de Ziguinchor sont relativement pauvres en azote $(\mathrm{N})$ qui résulte de la biodégradation de la MO.

L'ensemble des vallées a une teneur en $\mathrm{N}$ comprise dans l'intervalle [0,10-0,25\%]. Ainsi, les vallées sont considérées très pauvre en azote quel que soit le niveau de toposéquence. Cependant, le rapport $\mathrm{C} / \mathrm{N}$ est, en moyenne, inférieur à 12 dans toutes les vallées ; à l'exception de la partie aval de la vallée de Lyndiane. 
Tableau 3 : Variabilité de la teneur des éléments fertilisant, de la Capacité d'échange cationiques (CEC) et du Taux de saturation (TS) des so

\begin{tabular}{|c|c|c|c|c|c|c|c|c|c|c|c|c|}
\hline Vallée & Partie & $\% \mathrm{C}$ & MO \% & $\mathbf{N} \%$ & $\mathrm{C} / \mathrm{N}$ & $\mathbf{P}$ ppm & $\begin{array}{c}\mathrm{Ca} \\
\mathrm{meq} / 100 \mathrm{~g}\end{array}$ & $\underset{\mathrm{meq} / 100 \mathrm{~g}}{\mathrm{Mg}}$ & $\begin{array}{c}\mathrm{Na} \\
\mathrm{meq} / 100 \\
\mathrm{~g} \\
\end{array}$ & $\begin{array}{c}\mathrm{K} \\
\mathrm{meq} / 100 \\
\mathrm{~g} \\
\end{array}$ & $\begin{array}{c}\text { CEC } \\
\text { meq/100 } \\
\text { g } \\
\end{array}$ & $\% \mathrm{TS}$ \\
\hline \multirow{4}{*}{$\begin{array}{l}\text { Cobitène- } \\
\text { Colobane }\end{array}$} & Amont & 0,532 & 0,917 & 0,046 & 11,511 & 0,469 & 6,38 & 1,05 & 1,41 & 0,29 & 15,00 & 60,82 \\
\hline & Médiane & 0,158 & 0,272 & 0,014 & 11,255 & 3,158 & 2,33 & 1,43 & 1,04 & 0,61 & 32,00 & 16,86 \\
\hline & Aval & 0,098 & 0,170 & 0,008 & 11,724 & 0,811 & 1,50 & 1,73 & 0,91 & 0,32 & 32,00 & 13,93 \\
\hline & Moyenne & 0,263 & 0,453 & 0,023 & 11,497 & 1,480 & 3,40 & 1,40 & 1,12 & 0,41 & 26,33 & 30,54 \\
\hline \multirow[t]{4}{*}{ Diéfaye } & Amont & 0,355 & 0,611 & 0,031 & 11,511 & 1,067 & 3,38 & 0,53 & 0,77 & 0,24 & 26,00 & 18,86 \\
\hline & Médiane & 0,217 & 0,374 & 0,018 & 11,905 & 0,768 & 2,93 & 0,45 & 0,87 & 0,20 & 25,00 & 17,78 \\
\hline & Aval & 0,808 & 1,392 & 0,070 & 11,537 & 0,213 & 5,93 & 3,68 & 2,58 & 0,86 & 31,00 & 42,06 \\
\hline & Moyenne & 0,460 & 0,792 & 0,040 & 11,651 & 0,683 & 4,08 & 1,55 & 1,41 & 0,43 & 27,33 & 26,23 \\
\hline \multirow{4}{*}{$\begin{array}{l}\text { Kandialang- } \\
\text { Djibock }\end{array}$} & Amont & 0,453 & 0,781 & 0,039 & 11,557 & 4,439 & 5,25 & 0,68 & 0,40 & 0,16 & 16,00 & 40,56 \\
\hline & Médiane & 1,044 & 1,800 & 0,090 & 11,651 & 0,896 & 5,55 & 0,38 & 0,33 & 0,11 & 20,00 & 31,81 \\
\hline & Aval & 0,492 & 0,849 & 0,042 & 11,724 & 2,732 & 8,55 & 1,88 & 0,11 & 0,15 & 6,00 & 178,01 \\
\hline & Moyenne & 0,663 & 1,143 & $\mathbf{0 , 0 5 7}$ & 11,644 & 2,689 & 6,45 & 0,98 & 0,28 & 0,14 & 14,00 & 83,46 \\
\hline \multirow[t]{4}{*}{ Lyndiane } & Amont & 0,748 & 1,290 & 0,070 & 10,693 & 0,982 & 5,03 & 0,60 & 0,27 & 0,19 & 18,00 & 33,78 \\
\hline & Médiane & 0,433 & 0,747 & 0,042 & 10,317 & 1,024 & 1,35 & 0,15 & 0,11 & 0,11 & 32,00 & 5,36 \\
\hline & Aval & 0,532 & 0,917 & 0,042 & 12,662 & 0,854 & 9,00 & 0,83 & 0,10 & 0,11 & 32,00 & 31,37 \\
\hline & Moyenne & 0,571 & 0,985 & 0,051 & 11,224 & 0,953 & 5,13 & 0,53 & 0,16 & 0,14 & 27,33 & 23,50 \\
\hline \multirow{2}{*}{$\begin{array}{l}\text { Test de Kruskal- } \\
\text { Walli }\end{array}$} & $\mathrm{P}$-value & 0,408 & 0,408 & 0,431 & 0,622 & 0,376 & 0,64 & 0,47 & 0,03 & 0,04 & 0,25 & 0,44 \\
\hline & Alpha & 0,050 & 0,050 & 0,050 & 0,050 & 0,050 & 0,05 & 0,05 & 0,05 & 0,05 & 0,05 & 0,05 \\
\hline
\end{tabular}

$\boldsymbol{C}=$ Carbone, $\boldsymbol{M O}=$ Matière organique, $\boldsymbol{N}=$ Azote, $\boldsymbol{P}=$ Phosphore, $\boldsymbol{C a}=$ Calcium, $\boldsymbol{M g}=$ Magnésium, $\boldsymbol{N a}=$ Sodium, $\boldsymbol{K}=$ Potassium, $\boldsymbol{C E C}=$ Capacité

d'échange cationique, $\boldsymbol{T S}=$ Taux de saturation, $\mathbf{p p m}=$ partie pour mil, $\mathbf{m e q}=$ milliéquivalent, $\mathbf{g}=$ gramme. 
A l'instar de l'élément azote, les sols analysés sont très pauvres en phosphore. Aucune des vallées n'a atteint la teneur moyenne de 5 ppm en phosphore qui est estimée basse. La teneur la plus élevée en phosphore est estimée à 4,439 ppm et n'a pas été notée en amont de la vallée de KandialangDjibock.

L’analyse comparative a montré une différence significative de la variation de la teneur moyenne en $\mathrm{Na}$ et $\mathrm{K}$ suivant les vallées. Elle n'est cependant pas significative pour la variation des teneurs moyennes en $\mathrm{Ca}, \mathrm{Mg}$, de la CEC et du TS.

Les sols de la vallée de Cobitène-Colobane présentent la plus faible teneur moyenne en $\mathrm{Ca}(3,40 \mathrm{meq} / 100 \mathrm{~g})$. La teneur observée en amont représente environ 3 à 4 fois celle notée dans sa partie médiane et en aval. Elle est, en moyenne, estimée à 4,08 meq/100g dans la vallée de Diéfaye et est plus importante dans sa partie aval (5,93 meq/100g). Dans la vallée de Lyndiane, la teneur moyenne en Ca est plus élevée en aval $(9,00 \mathrm{meq} / 100 \mathrm{~g})$ et dans la partie médiane. La vallée de Kandialang-Djibock présente la plus importante teneur moyenne en Ca. Elle est plus importante dans la partie aval $(8,55$ meq/100g) comparée à celle estimée à $5,25 \mathrm{meq} / 100 \mathrm{~g}$ et $5,55 \mathrm{meq} / 100 \mathrm{~g}$ respectivement dans les parties amont et aval. Dans l'ensemble, les teneurs en Ca dans toutes les vallées étudiées sont supérieures à 0,2 meq/100g. La teneur en élément $\mathrm{Mg}$ est très élevée ([Mg] >0,5 meq/100g) dans les sols de l'ensemble des vallées de Ziguinchor. Cependant, la plus importante teneur en Mg est enregistrée en aval de ces vallées.

L'analyse de la teneur en sodium montre, de manière générale, que les vallées de Diéfaye et Cobitène-Colobane sont sodiques ([Na] >1meq/100g) alors que celles de Kandialang-Djibock et Lyndiane ne le sont pas ([Na] < 1 meq/100g). La teneur en $\mathrm{Na}$ est quasi équitablement répartie suivant le gradient de toposéquence dans la vallée de Cobitène-Colobane. Par contre, dans celle de Diéfaye, une plus grande concentration de Na est enregistrée en aval (2,58 meq /100g). Elle constitue presque le triple de celle notée dans sa partie médiane et en amont.

D’une manière globale, les sols des vallées de Ziguinchor présentent une réponse relativement probable aux engrais potassiques $([\mathrm{K}]<0,5$ meq/100g). Cependant, en amont de la vallée de Cobitène-Colobane et en aval de la vallée de Diéfaye, la réponse aux engrais potassiques n'est pas probable. Elles présentent, respectivement, une teneur en $\mathrm{K}$ de $0,61 \mathrm{meq} / 100 \mathrm{~g}$ et 0,86 meq/100g.

La valeur moyenne de la CEC des sols dans les vallées de CobitèneColobane (26,33 meq/100g), Diéfaye (27,33 meq/100g) et Lyndiane $(27,33$ meq/100g) est considérée haute.

Le pourcentage des cations nutritifs (TS) parmi les bases échangeables est estimé moyen dans les vallées de Lyndiane (23,50\%), Diéfaye (26,23\%) et 
celle de Cobitène-Colobane (30,54\%). Il est cependant élevé dans la vallée de Kandialang-Djibock $(83,46)$. Le TS de la vallée de KandialangDjibock dépasse $80 \%$.

\section{Corrélations des composantes physico-chimiques des sols}

Le test de sphéricité de Bartlett, a permis de déterminer une corrélation significative $(\mathrm{p}<0,01)$ entre les variables physico-chimiques des sols. La teneur en sable est négativement corrélée à celles de l'argile, du limon et du potassium $(\mathrm{K})$; avec des coefficients de corrélation respectifs de 0,793 ; -0,736 et $-0,590$. Par ailleurs, la corrélation est fortement négative entre le pH et la CEC ( $\mathrm{r}=-0,998)$ d'une part, et entre la CEC et le taux de saturation du sol $(r=-0,776)$ d'autre part. On note, cependant, une corrélation hautement positive entre la teneur en azote des sols et celle du carbone $(\mathrm{r}=$ 0,995), d'une part, et entre la teneur en azote et celle de la MO ( $\mathrm{r}=0,995)$, d'autre part.

La Figure 3 correspond à la régression linéaire de la variation de la $\mathrm{CEC}$ et celle de $\mathrm{N} \%$, respectivement, en fonction du $\mathrm{pH}$ et du Carbone (\%).

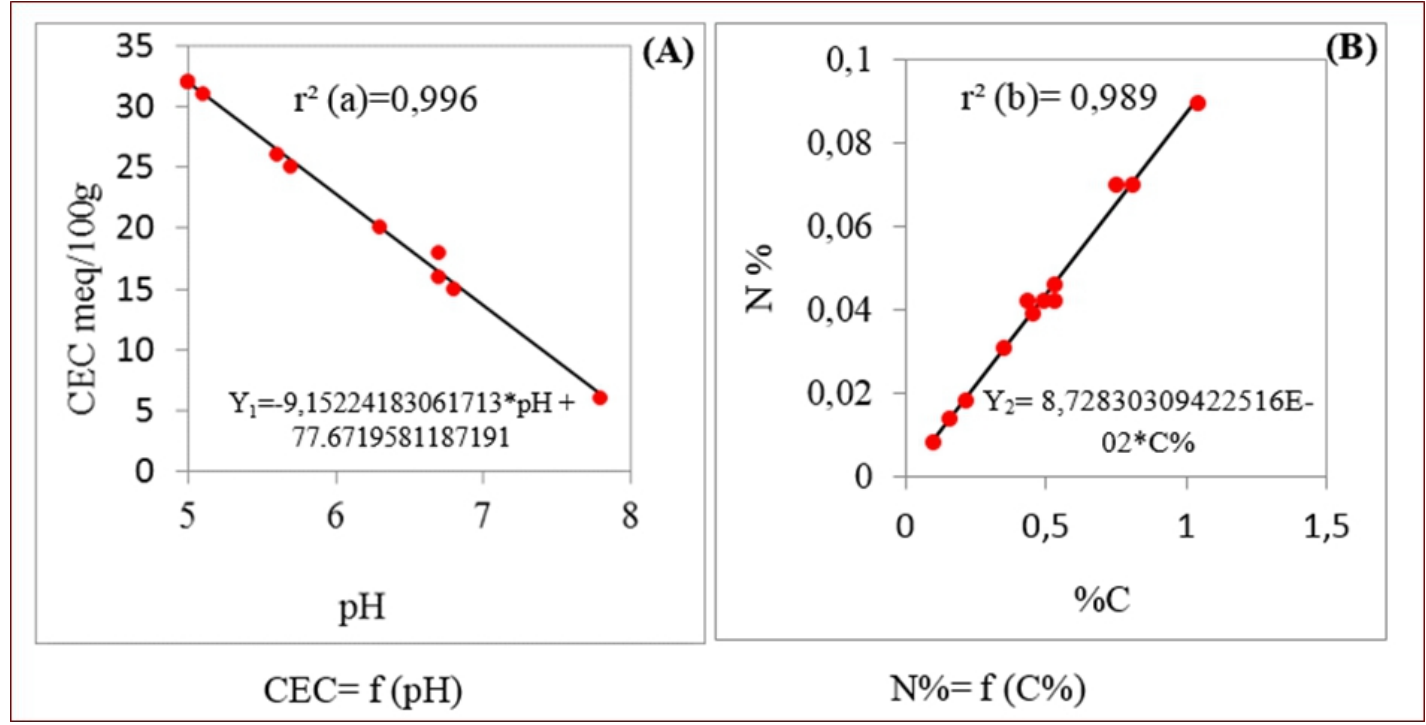

Figure 3 : Régressions linéaires de la $\mathrm{CEC}$ en fonction du $\mathrm{pH}(\mathrm{A})$ et de la teneur en $\mathrm{N}$ en fonction du C (B) dans les sols des vallées.

Les coefficients de détermination $\left(\mathrm{r}^{2}\right)$ de ces deux régressions linéaires sont élevés. La régression $\mathrm{Y}_{1}$ avec $\mathrm{r}^{2}=99,6 \%$ assure une estimation correcte de la CEC des sols analysés en fonction de leurs $\mathrm{pH}$. La régression $\mathrm{Y}_{2}$ avec $\mathrm{r}^{2}=$ $98,9 \%$ donne aussi une bonne estimation de la teneur en azote $(\mathrm{N})$ des sols en fonction du pourcentage de carbone.

Le $\mathrm{pH}$ est positivement corrélé au taux de saturation du sol $(\mathrm{r}=0,767)$. Ce qui signifie que, plus le $\mathrm{pH}$ du sol est basique, plus le pourcentage des 
cations disponibles pour les cultures est élevé. La CE est positivement corrélée à la teneur en $\mathrm{Mg}(\mathrm{r}=0,830), \mathrm{Na}(\mathrm{r}=0,882)$ et $\mathrm{K}(\mathrm{r}=0,848)$. Ce qui explique que la conductivité électrique du sol augmente avec la concentration en ions $\mathrm{Mg}^{+}, \mathrm{Na}^{+}$et $\mathrm{K}^{+}$. Ces ions contribuent à la salinisation des sols. La teneur en Mg est positivement corrélée à celle de $\mathrm{Na}(\mathrm{r}=0,752)$ et de $\mathrm{K}(\mathrm{r}=0,822)$. Par ailleurs, la corrélation entre la teneur des sols en K et celle de $\mathrm{Na}$ est hautement positive. Il est important de noter que ces différentes variables chimiques sont interdépendantes et varient proportionnellement dans le sol.

\section{Analyse comparative des composantes principales des sols en fonction des vallées}

La Figure 4 présente la carte factorielle de l'ACP réalisée sur les composantes physico-chimiques des sols des vallées agricoles de la ville de Ziguinchor. L'analyse de ce graphique a permis de noter 4 groupes discriminant. Le groupe $\mathrm{G} 1$ est représenté par les variables $\mathrm{MO} \%, \mathrm{~N} \%, \mathrm{C} \%$, $\mathrm{pH}$ et $\mathrm{TS} \%$ qui sont positivement corrélées à l'axe F2 et aux échantillons de sol des vallées de Kandialang-Djibobok dans les parties amont (KA) médiane (KM) et aval (Kav). Cette corrélation positive est également observée dans les parties amont de Cobitène-Colobane (CA) et Lyndiane (LA). Ainsi, les principales caractéristiques de ces vallées sont liées aux teneurs en $\mathrm{MO} \%$, $\mathrm{N} \%, \mathrm{C} \%, \mathrm{pH}$ et au TS\%. La partie amont de la vallée de Cobitène-Colobane (CA) est fortement corrélée aux variables $\mathrm{MO} \%, \mathrm{C} \%$ et $\mathrm{N} \%$. Ce résultat montre que la partie amont de cette vallée est très pourvue en MO. Cependant, les composantes du groupe G1 sont négativement corrélées à la CEC (groupe G3). Ce qui traduit que les sols de la vallée de Kandialang-Djibobok et ceux de la partie amont de la vallée Cobitène-Colobane et celle de Lyndiane sont peu fournis en bases échangeables $\left(\mathrm{K}^{+}, \mathrm{Ca}^{2+}, \mathrm{Mg}^{2+}, \mathrm{Na}^{+}\right)$et vice versa. 


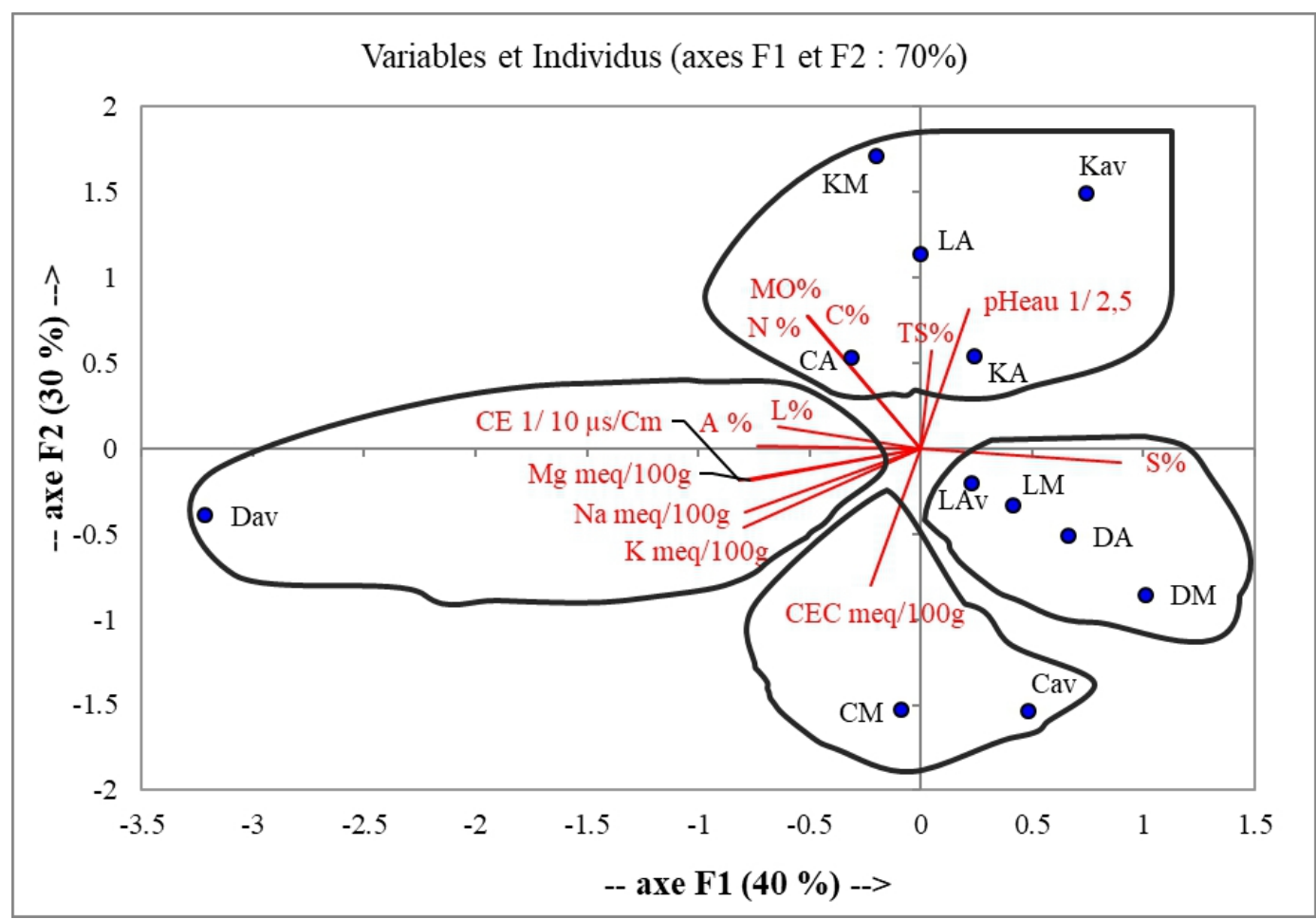

Figure 4 : L'ACP des variables caractéristiques des sols des vallées agricoles de la ville Ziguinchor

Le groupe G2 est représenté par la variable S\% qui est positivement corrélée à l'axe F1 et aux échantillons de sols des parties amont (DA) et médiane (DM) de la vallée de Diéfaye, ainsi que ceux des parties médiane (LM) et aval (LAv) de la vallée de Lyndiane. Ce qui traduit, en termes de granulométrie, la prédominance de la fraction sable dans ces parties. Les facteurs du groupe G2 sont négativement corrélés à ceux du groupe G4 sur l'axe F1. Ce qui exprime la faible concentration des facteurs du groupe G4 dans les sols des parties amont et médiane de la vallée de Diéfaye, ainsi que ceux des parties médiane et aval de la vallée de Lyndiane. Le groupe G3 correspond au facteur CEC qui est négativement corrélé à l'axe F2 et aux sols de partie médiane $(\mathrm{CM})$ et celle aval (CAv) de la vallée de Cobitène-Colobane. Il en ressort que la partie médiane et celle aval de la vallée de CobitèneColobane sont beaucoup plus pourvues en bases échangeables par opposition à celle du groupe G1. La position du groupe G4 constitué du singleton (DAV) de la partie aval de la vallée de Diéfaye, est lié aux facteurs L\%, A\%, CE, Mg, $\mathrm{Na}$ et K qui sont négativement corrélés à l'axe F1. 


\section{Travail de sol et leur fertilization}

Deux techniques principales de labour du sol sont notées dans le système rizicole. Il s'agit du labour en billon dans $66,67 \%$ des cas. Les billons de 15 à $20 \mathrm{~cm}$ d'épaisseur et 20 à $30 \mathrm{~cm}$ de largeur sont dressés perpendiculairement au sens d'écoulement de l'eau de surface. L'autre technique de préparation du substrat, pratiqué dans 33, 33\% des cas, se rapporte au labour à plat du sol ; à une profondeur d'environ 20 à $30 \mathrm{~cm}$. Elle a été principalement notée dans la partie amont des vallées de KandialangDjibock et de Diéfaye, dans les casiers où la lame d'eau est généralement faible.

La fertilisation des sols est essentiellement basée sur l'apport de matière organique et la gestion des résidus de cultures. L'épandage de la fumure organique (ordures ménagères et bouse animale) dans les casiers rizicoles constitue le principal mode de fertilisation des sols. Cette étude a permis de noter que seuls $2,78 \%$ des producteurs utilisent, à faible dose, les engrais minéraux. Les $80,56 \%$ des riziculteurs épandent la fumure organique au moment du labour.

\section{Discussion}

La discussion des résultats porte particulièrement sur les caractéristiques physicochimiques des sols des vallées agricoles de Ziguinchor. Elle concerne également les potentialités agronomiques de ces sols et leurs contraintes majeures de production agricole liées à la texture sableuse et au caractère acido-salin de certaines parties.

\section{Caractères texturaux et granulométriques des sols}

Les ressources pédologiques de l'horizon superficiel $(0-20 \mathrm{~cm})$ des vallées agricoles périurbaines de Ziguinchor présentent, du point de vue de leurs propriétés physiques, une texture relativement équilibrée, de nature principalement limono-sablo-argileuse. Elles ont une structure relativement stable à caractère hydromorphe, en raison de leur bonne capacité de rétention hydrique. En effet, toutes les trois fractions granulométriques sont d'une manière générale bien représentées. Ce qui leur confère de bonnes potentialités agronomiques surtout pour la production rizicole. Ces types de sols composés de 50 à $70 \%$ de sable, 30 à $50 \%$ de limon et 15 à $25 \%$ d'argile ont déjà été notés par Tossou et al. (2006) dans les vallées agricoles de la conurbation de Abomey-Bohicon au Bénin.

Cependant, certaines parties de ces vallées sont, du point de vue granulométrique, prédominées par la fraction sable. Cet état de fait pourrait être expliqué, en partie, par l'érosion hydrique observé au niveau du plateau et l'ensablement progressif des casiers rizicoles. Ce phénomène est beaucoup plus accentué dans les vallées de Kandialang-Djibock et Lyndiane qui 
présentent la plus forte teneur en sable en aval, respectivement estimée à $66,38 \%$ et $48,63 \%$. En effet, selon Manzelli et al. (2015), l'ensablement représente une problématique majeure de la riziculture en Casamance. Le processus d'ensablement est en train de modifier l'état de la structure des sols des vallées agricoles de la ville de Ziguinchor, qui du point de vue général, était quasi stable, fragmentaire et propice à la production agricole (Dasylva, 2018). La vallée de Kandialang-Colobane constitue une zone de convergence des eaux de ruissellement du plateau qui polarise les quartiers de Tilène, Kandé et Kandialang. La vallée de Lyndiane quant à elle, collecte les eaux de ruissellement provenant du plateau de Boucotte Sud et Lyndiane. Ces quartiers font d'ailleurs partie de ceux caractérisés par une faible densité du réseau routier butiné et un couvert végétal peu important. L'effet splash des pluies intenses souvent enregistrées dans la ville de Ziguinchor favorise donc le décapage du sol, le transport des particules sableuses par les eaux de ruissellement et l'ensablement des rizières. En tout état de cause, le phénomène d'ensablement des casiers rizicoles associé à l'acidification et à la salinisation des terres dans les vallées est en train de compromettre la riziculture dans la ville de Ziguinchor.

\section{Caractères acido-alcalin : facteurs limitant de la production agricole}

Le caractère acide des sols des bas-fonds constitue l'un des principaux facteurs limitant de la production agricole dans la ville de Ziguinchor. Il est beaucoup plus manifeste dans la partie médiane et aval des vallées de Cobitène-Colobane et Lyndiane ; ainsi qu'en aval de celle de Diéfaye où le pH est environ égal à 5.

La vallée de Kandialang-Djibock présente les meilleures caractéristiques chimiques en raison de son $\mathrm{pH}$ relativement neutre d'amont en aval et qui est bien adapté à la plupart des cultures (Doucet, 2006). Dans la plupart des casiers rizicoles exploités, la salinité des sols n'a pas atteint un seuil de nuisibilité aux cultures. Celui-ci est, selon Durand (1983) et Grûnberge (2015), supérieur à $500 \mu \mathrm{S} / \mathrm{cm}$. Cependant, force est de noter que l'effet conjugué de l'acidité et de la salinité a rendu inexploitable $8,73 \%$ des 838,9 ha rizicoles de la commune de Ziguinchor (Dasylva et al., 2017). Cet état de fait a été beaucoup plus noté dans la vallée de Diéfaye et celle de Cobitène-Colobane qui ont, d'ailleurs, enregistré les concentrations du sol en $\mathrm{Na}$ les plus élevées, respectivement estimées à $1,12 \mathrm{meq} / 100 \mathrm{~g}$ et 1,41 meq/100g. Dans ces casiers rizicoles abandonnés, la conductivité électrique évaluée $3999 \mu \mathrm{S} / \mathrm{cm}$ a largement dépassé le seuil critique de résistance du riz au stress salin qui a été évalué à $3000 \mu \mathrm{S} / \mathrm{cm}$ par Zongo (2014). En effet, la particularité du caractère sodique des sols de ces deux vallées résulte de l'influence des eaux salées du fleuve Casamance. Dans ces deux vallées, l'avancée de la langue salée dans les casiers rizicoles frontaux au fleuve 
Casamance est accentuée par la dégradation des digues anti-sel. Ces digues anti-sel, autrefois construites par les paysans diolas, ne sont plus entretenues. Par ailleurs, la bande de mangrove qui protégeait ces digues s'est dégradée en raison de l'effet conjugué de la pression anthropique et des péjorations climatiques notées par divers auteurs (Marius , 1982 ; Montoroi, 1998 ; Tendeng et al., 2016).

\section{Teneur en éléments fertilisant des sols}

Les résultats de l'analyse des sols ont permis de noter une relative richesse des vallées agricoles de la ville de Ziguinchor en matière organique. Ce qui se justifie, en partie, par le fait que la majorité des producteurs $(80,56 \%)$ procèdent à l'épandage de fumier organique dans les casiers rizicoles et à l'enfouissement des résidus agricoles pendant le labour du sol. Ces pratiques culturales ont un double objectif. Elles visent à maintenir le niveau de fertilité des sols et de lutter contre leur acidification (Dasylva, 2018). La présence de la végétation ligneuse dans les vallées contribue, par ailleurs, à améliorer la fertilité des sols. Plus de $28 \%$ des ménages agricoles de la commune de Ziguinchor ont déclaré, à ce titre, que la fonction d'amélioration de la fertilité des sols serait l'une des principales raisons qui justifient le choix délibéré de laisser la végétation ligneuse dans ces bas-fonds (Dasylva et $a l .$, 2017). Les vallées de Kandialang-Djibock et Lyndiane ont présenté la plus importante teneur en MO respectivement évaluée en moyenne à $1,143 \%$ et $0,985 \%$. En se basant sur les travaux du CIRAD et GRET (2002), ces vallées pourront être considérées comme étant très riches en MO. Ces deux vallées, comparées à celles de Diéfaye et Cobitène-Colobane, sont caractérisées par une meilleure intégration du couvert végétal principalement constitué de l'espèce $E$. guineensis. Cette végétation contribue de manière significative à l'apport de la $\mathrm{MO}$ issue de la décomposition de la litière. Par ailleurs, la richesse en MO de la vallée de Kandialang-Djibock aurait influencé positivement l'augmentation du $\mathrm{pH}$ de ces sols. Celui-ci est relativement neutre sur toute la toposéquence de la vallée. Ce qui confirme les travaux de Diallo et al. (2015) qui ont montré que la décomposition de la litière végétale contribue à l'augmentation de la valeur du $\mathrm{pH}$ et à l'amélioration de la fertilité des sols. Cette étude a d'ailleurs montré que le $\mathrm{pH}$ du sol est corrélé à 76,7\% à son taux de saturation. Ce qui montre que ces deux paramètres varient proportionnellement dans le sol et traduisent ainsi que les sols basiques sont plus riches en cations disponibles pour les cultures, comparés à ceux acides. A la lumière des travaux de Soltner (1992), on pourrait noter, d'une manière globale, que les sols des vallées agricoles de la ville de Ziguinchor sont oligo-saturés en raison de leur taux de saturation en bases échangeables qui dépasse rarement 30\% à l'exception de la vallée de Kandialang-Djibock. Les sols de cette vallée ont un taux de saturation en 
bases échangeables estimé, en moyenne, à 83,46\%. Ce qui leur confêre le caractère de sols limono-sablo-argileux saturés en éléments nutritifs disponibles pour les cultures. On peut déduire, de ce résultat, que les réserves calciques de cette vallée sont suffisantes et que le fonctionnement physicochimique et biologique de son sol est optimal.

Bien que les sols analysés soient relativement riches en MO, il faut noter que l'azote et le phosphore ont été estimés très pauvres dans l'ensemble de ces vallées. Leur teneur moyenne respective varie entre 0,057\% et 2,689 $\mathrm{ppm}$. Pourtant, le rapport $\mathrm{C} / \mathrm{N}$ a partout donné des valeurs inferieures à 12 , démontrant ainsi une intense activité biologique dans ces sols, un bon processus de minéralisation de la $\mathrm{MO}$ et par conséquent, une bonne libéralisation de la quantité d'azote dans le sol. Cette étude a, d'ailleurs, permis de noter que la teneur en azote des sols est liée, à 99,5\%, à celle de la MO. Ce qui traduit que la concentration en azote des sols est fortement dépendante de leur richesse en MO et de leurs activités biologiques. Ce qui corrobore l'hypothèse selon laquelle l'azote nécessaire à la croissance des cultures relève de la biodégradation de la $\mathrm{MO}$ du sol. Cette dichotomie de la teneur en $\mathrm{MO}$ et de l'azote, ainsi que la pauvreté des sols en phosphore pourraient s'expliquer par le fait que ces éléments nutritifs soient prélevés par les cultures ou par la faible abondance des bio-réducteurs dans le milieu. D'ailleurs, le fait que les vallées de la commune de Ziguinchor soient toujours exploitées toute l'année est la raison fondamentale que les carottes de sols soient prélevées pendant la période de récolte. En ce moment, les cultures auraient déjà prélevé la quantité importante d'azote, nécessaire à leur croissance. Il en est de même pour le phosphore qui assure la multiplication des cellules, la respiration photosynthétique et la croissance du système racinaire comme indiqué par UNIFA (2012 et 2014).

Il est par ailleurs indispensable de mentionner que cette étude a permis de noter que la proportion de la fraction sableuse est négativement corrélée à la teneur en potassium des sols. Ce résultat montre que plus un sol est sableux, moins il est pourvu en potassium (K). Ce qui confirme que l'ensablement contribue à la baisse de la fertilité des sols en potassium utile à la fructification et à la maturation des cultures comme l'a démontré Le Mire (2012). Par ailleurs, la corrélation est fortement négative entre le $\mathrm{pH}$ et la CEC $(\mathrm{r}=$ $0,998)$ d'une part, et entre la CEC et le taux de saturation du sol $(r=-0,776)$ d'autre part. Ce qui traduit que la CEC du sol est inversement proportionnel à son $\mathrm{pH}$ et à sa teneur en cations nutritifs $\left(\mathrm{K}^{+}, \mathrm{Ca}^{2+}, \mathrm{Mg}^{2+}, \mathrm{Na}^{+}\right)$.

\section{Conclusion}

Cette étude a permis de noter, d'une manière générale, une prédominance de sols hydromorphes à texture limono-argilo-sableuse, caractérisés par une structure relativement stable. Ces caractères physiques 
leur confèrent de grandes potentialités agronomiques, surtout pour la production rizicole. Par ailleurs, les pratiques de fertilisation consistant à l'épandage de fumier dans les casiers rizicoles (observés chez 80,56\% des agriculteurs), à l'enfouissement des résidus des cultures confèrent à ces sols une relative richesse en matière organique et, par conséquent, en élément nutritifs disponibles pour les cultures. Il s'y ajoute les aménagements liés à l'établissement des diguettes et des digues hydro-agricoles. Ces pratiques contribuent au maintien de la fertilité des sols et à l'amélioration des rendements agricoles.

Les principaux facteurs qui sont en train d'altérer la qualité des sols et d'entraver la production agricole dans ces bas-fonds sont, entre autres, l'ensablement, l'acidification conjuguée à la salinisation progressive des casiers rizicoles. Outre les 73,24 ha devenus impropres à la production agricole, à cause de l'effet conjugué de l'acidité et de la salinité dans les vallées de Diéfaye et Cobitène-Colobane, l'ensablement est en train de modifier progressivement la texture et la structure du substrat des bas-fonds de la commune de Ziguinchor. Il s'y ajoute l'urbanisation galopante de la commune de Ziguinchor qui se traduit par l'occupation anarchique et progressive des vallées par le bâti. Ce qui laisse présager d'un problème de viabilité de l'agriculture urbaine dans la commune de Ziguinchor.

Toutefois, la réhabilitation des digues anti-sel dans les vallées de Cobitène-Colobane et Diéfaye, devrait permettre d'atténuer les effets de la salinisation. La problématique de l'ensablement des vallées pourrait être résorbée par l'établissement des canaux et des égouts d'évacuation des eaux de ruissellement dans les quartiers périurbains situés en zone de plateau de la ville tels que Kansahoudy, Boucotte sud, Lyndiane, Perisack, Tilène et Kandé. Entre autres contraintes, l'occupation de ces vallées non aedificandi, doit être résolue sur la base d'un arrêté municipal d'interdiction promu et connu de tous les acteurs au développement de la commune.

\section{References:}

1. ANSD, 2015. - Projection de la population du Sénégal, 2013-2063. NSD/MEFP, $175 \mathrm{p}$.

2. Aubry, C. 2012. -Multifonctionnalités de l'agriculture urbaine : Acquis et questions dans les pays du Nord et du Sud. In : conférence à l'HEPIA-Genève, le 11 septembre 2012.

3. Ba A., Cantoreggi N., Simos J. et Duchemin E. 2016. - Impacts sur la santé des pratiques des agriculteurs urbains à Dakar (Sénégal)», VertigO - la revue électronique en sciences de l'environnement [En ligne], Volume 16 Numéro 1 | mai 2016, mis en ligne le 09 mai 2016, consulté le 02 juillet 2017. URL : http://vertigo.revues.org/17030 ; DOI : 10.4000/vertigo.17030. 
4. Bagré A. S., Kientéga M., Cissé G. et Tanner M., 2002. -Processus de reconnaissance et de législation de l'agriculture urbaine à Ouagadougou. De la légitimation à la légalisation. Rev. inter. sci. de la terre, $\mathrm{N}^{\circ}$ spécial, $10 \mathrm{p}$.

5. INP, 1992.- Méthodes d'analyses des sols : manuel pratique, $29 \mathrm{p}$.

6. CIRARD et GRET, 2002.- Mémento de l'agronome, Ministère des Affaires Etrangères, France, 1700 p.

7. Dasylva M., 2013. - Agriculture urbaine sous pluie et sécurité alimentaire dans la commune de Ziguinchor. Mémoire de Master, Département d'agroforesterie, UFR-ST, UASZ, 51 p.

8. Dasylva M, Ndour N, Ndiaye O, Sambou B. 2017.- Analyse de la flore, de la végétation ligneuse et des fonctions des vallées en zone périurbaine post-conflit (Ziguinchor, Sénégal). Int. J. Biol. Chem. Sci. 11(1) : 360-377. DOI : http://dx.doi.org/10.4314/ijbcs.v11i1.28.

9. Dasylva M., 2018. - Caractérisation et analyse de l'agriculture intra et périurbaine dans le commune de Ziguinchor ; Thèse de Doctorat en Science agronomique et environnement, Université Assane Seck de Ziguinchor, $262 \mathrm{p}$.

10. Dasylva M, Ndour N, Sambou B, Soulard C-T. 2018. -Les microexploitations agricoles de plantes aromatiques et médicinales : élément marquant de l'agriculture urbaine à Ziguinchor, Sénégal. Cah. Agric. 27 : 25004. 9 p. DOI : https://doi.org/10.1051/cagri/2018011

11. Doucet R. 2006. - Le climat et les sols agricoles. Ed. Berger, Eastman, Québec. xv, 443 p.

12. Diallo M. D., Saleh M. M., Ndiaye O., Diop A., Guissé A., 2015.Influence de la décomposition de la nécro-masse des espèces végétales tropicales sur le $\mathrm{pH}$ et la structure génétique des communautés bactériennes d'un sol ferrugineux tropical au Sénégal, Journal of Applied Biosciences 91:8547 - 8558, ISSN 1997-5902

13. Duchemin E., 2012. - Agriculture urbaine : quelle définition ? une actualisation nécessaire?, [En ligne], Mise en ligne le 14 décembre 2012, consulté le 23 juillet 2017, URL :https://agriurbain.hypotheses.org/2705.

14. Durand J.H., 1983. - Les sols irrigables, Etude pédologique. Ed. Département de géographie de l'université de Paris - Sorbonne, $389 \mathrm{p}$ Durr C.1984.- Systèmes de culture et élaboration du rendement du riz (Oryza sativa L.) en Camargue. Thèse de Doctorat. Paris. INA P-G. $195 \mathrm{p}$.

15. FAO-UNESCO, 1975. - mondiale des sols au $1: 5000$ 000eme , volume 1, légende, $68 \mathrm{p}$.

16. FAO. 2010. -Agriculture intelligente face au climat, Food and Agriculture

Organization. 
http://www.fao.org/climatechange/climatesmart/66250/fr/. [Consulté le 24 février 2011].

17. Faraoun F. et Bénabdèli K., 2010.- Cartographie et caractérisation physico-chimique des sols de la plaine de Sidi Bel Abbés (Algérie occidentale), Afrique SCIENCE 06(3), ISSN 1813-548X, http://www.afriquescience.info, pp $18-26$.

18. Grûnberge O., 2015.- Dynamiques salines des sols des milieux arides et semi-arides. Sciences de la Terre. Université de Montpellier, LISAH - Laboratoire d'étude des interactions entre sols, agrosystèmes et hydrosystèmes <tel-01140309>

19. Hista J., 2007.- Enjeux autour de l'agriculture urbaine contemporaine, Regroupement des Jardins Collectifs du Québec, $12 \mathrm{p}$

20. Kakai HF, Kakai AG et Tohouegnon AG., 2010. - Agriculture urbaine et valorisation des déchets au Bénin : une approche de développement durable », VertigO - la revue électronique en sciences de l'environnement [En ligne], Volume 10 numéro 2 | septembre 2010, mis en ligne le 29 septembre 2010, consulté le 11 août 2017. URL: http://vertigo.revues.org/9994 ; DOI : 10.4000/vertigo.9994

21. Keita A., 2013- La mutation des terres agricoles autour de Ziguinchor ; Mémoire de master ATDDL, FLSH, UCAD, 96 p.

22. Koné` B., Diatta S., Sylvester O., Gbalou Y., Camara M., Djidji D. D., et Assa A. 2008.- Estimation de la fertilité potentielle des ferrasols par la couleur, CANADIAN JOURNAL OF SOIL SCIENCE, pp 331342..- Etude du sol de la région d'Aigrefeuille-sur-Maine (LoireAtlantique) à partir de la base de données des analyses de terre (INRA) ; Donesol, $18 \mathrm{p}$

23. Landon, J.R., 1984.- Booker Tropical Soil Manual. xiv. Booker Agriculture International Ltd., London, and Longman, Burnt Mill, U.K, $450 \mathrm{p}$.

24. Le Mire G., 2012.- Etude de l'impact d'une fertilisation potassique sur les caractéristiques physico-chimiques de la banane dessert au cours de sa croissance et à maturité, Mémoire de Master FAGE (Biologie et Ecologie pour la Forêt, l'Agronomie et l'Environnement Spécialisation Biologie des Interactions Plante-Environnement), Université de Lorraine, 32 p.

25. Manzelli M., Fiorillo E., Bacci M., Tarchiani V. 2015.- La riziculture de bas-fond au sud du Sénégal (Moyenne Casamance): enjeux et perspectives pour la pérennisation des actions de réhabilitation et de mise en valeur, Cah Agric, vol. 24, nº 5, doi: 10.1684/agr.2015.0772, pp. 301-3012.

26. Marius C., 1982. -Acid sulphate soils of the mangrove area of Senegal and Gambia. In Dost H., Van Breemen N. (éd.). Acid sulphate soils. 
Proceedings of the international symposium on acid sulphate soilsb (Bangkok, Thailand, February 1981), Wageningen, The Netherlands, ILRI, Publ. 31, p. 103-136.

27. Montoroi JP. 1998.- La riziculture inondée en Basse-Casamance (Sénégal). Contribution des petits barrages antisel à la réhabilitation des bas-fonds chimiquement dégradés par la sécheresse In CheneauLoquay A., Leplaideur A. (édit.). Les rizicultures de l'Afrique de l'Ouest. Actes du colloque international Quel avenir pour les rizicultures de l'Afrique de l'Ouest ? 5-7 avril 1995, Bordeaux, France. Cirad, Montpellier, France, pp 304-316.

28. Masson J.C., 2012.- Etude du sol de la région d'Aigrefeuille-surMaine (Loire-Atlantique) à partir de la base de données des analyses de terre (INRA) ; Donesol, $18 \mathrm{p}$.

29. PAM, 2012. -Rapport d'évaluation de la sécurité alimentaire en milieu urbain; communes de Kaolack, Kolda, Tambacounda et Ziguinchor; Rapport national du Sénégal; 24 p.

30. Rafik F., Saber N., Zaakour F., Mohcine H., Moustarhfer K. et Marrakchi C., 2015.- Caractérisation physico-chimique et estimation de la stabilité structurale des sols agricoles de la région Sidi Rahal, Sahel (Chaouia Cotiere, Maroc), European Scientific Journal, vol.11, No.27 ISSN: 1857 - 7881 (Print) e - ISSN 1857- 7431, pp 48-63.

31. Ramade F., 2008.- Dictionnaire encyclopédique des sciences de la nature et de la biodiversité Ed. Dunod, Paris, ISBN 978-2-10-053670$2,737 \mathrm{p}$.

32. Reyniers F.N., Truong, B. Bois J.F, Bonnin E. et Thomin G. 1979.Caractérisation de l'enracinement du riz pluvial in situ avec le phosphore-32, IAEA-SM-235144, pp 135-167

33. Sagna P., 2005. -Dynamique du climat et son évolution récente dans la partie ouest de l'Afrique occidentale; THESE DE DOCTORAT D'ETAT ES. LETTRES, UCAD ; Tome 1 et $2 ; 742$ p.

34. Soltner D., 1992.-Les bases de la production végétale. Tome 1 : le sol. Collection Sciences et Techniques Agricoles, 19ème édition, Sainte Gemmes sur Loire.

35. Sow D., 2014. -Analyse diachronique de la croissance spatiale de la ville de Ziguinchor de 1960 à 2014, Mémoire de Master, Département de Géographie, UASZ, 116 p

36. Sueur, M.J-P, 2011. -Villes du futur, futur des villes : quel avenir pour les villes du monde? (Analyses), Rapport d'information n ${ }^{\circ}$ 594, 353 p.

37. Tendeng M, Ndour N., Sambou B., Diatta M., Aouta A., 2016.Dynamique de la mangrove du marigot de Bignona autour du barrage d'Affiniam (Casamance, Sénégal), Int. J. Biol. Chem. Sci. 10(2): 666680, ISSN 1997-342X (Online), ISSN 1991-8631 (Print). 
38. Tossou R. C., Vodouhe S. D., Fanou J. A., Babadankpodji P. A., Kouevi a. T. et Aholoukpe H., 2006.- Caractéristiques physico chimiques et aptitudes culturales de sols de la conurbation AbomeyBohicon, Bénin ; UAC, Abomey-Calavi, FSA, document de travail Ecocité ${ }^{\circ} 9$, www.ecocite.org, 23 p.

39. Touhtouh D., Moujahid Y., El Faleh E.M., EL Halimi R., 2014.Caractérisations physicochimiques de trios types de sols du Sais, Maroc, J. Mater. Environ. Sci. 5 (5) (2014), ISSN : 2028-2508, CODEN : JMESCN, pp 1524-1534.

40. UNIFA. 2012.- Le Soufre, Indispensable à la synthèse des protéines, Fiche $\mathrm{N}^{\circ} 03$, www.unifa.fr, $06 \mathrm{p}$.

41. UNIFA., 2014.- Engrais azotés, Réduire les pertes, Augmenter leur efficacité, Fiche $\mathrm{N}^{\circ} 32$, www.unifa.fr ; 6 p.

42. Zongo N. E., 2014.- Effet de la salinité sur l'efficacité d'utilisation de l'azote parle riz, Mémoire de Master en Production Végétale, université polytechnique de Bobo-Dioulasso; institut du développement rural, $60 \mathrm{p}$. 